\section{Missing the Obvious}

\section{To the Editor:}

The article by Derot and colleagues ${ }^{1}$ describes a 60 -year-old woman with rheumatoid arthritis (RA) who developed 4 pulmonary nodules taking methotrexate (MTX) therapy ${ }^{1}$. MTX was discontinued. Because of uncontrolled synovitis, the patient was started on etanercept monotherapy. The authors documented marked improvement of the pulmonary nodules 21 months after the initiation of etanercept and further improvement later on. The article does not specify when exactly MTX was discontinued, but it appears this was not long before etanercept was started. The authors conclude that the etanercept likely led to shrinkage of the pulmonary nodules. In their discussion an obvious alternative conclusion was not mentioned: that the mere discontinuation of methotrexate led to the observed improvement in the pulmonary nodules. Marked improvement of rheumatoid nodules after discontinuation of MTX has been well documented ${ }^{2}$ and, in my own clinical experience, can be quite dramatic. Any inference about possi- ble clinical benefit for rheumatoid nodules related to etanercept therapy needs to be strongly cautioned.

DIRK M. NUENNINGHOFF, MD, MS, Dean Clinic, Rheumatology, 1313 Fish Hatchery Road, Madison, Wisconsin 53715, USA.

E-mail:nuenninghoff.dirk@tds.net

\section{REFERENCES}

1. Derot G, Marini-Portugal A, Maitre B, Claudepierre P. Marked regression of pulmonary nodules under etanercept therapy. J Rheumatol 2009;36:237-9

2. Kerstens PJ, Boerbooms AM, Jeurissen ME, Fast JH, Assmann KJ, van de Putte LB. Accelerated nodulosis during low dose methotrexate therapy for rheumatoid arthritis. An analysis of ten cases. J Rheumatol 1992;19:867-71

J Rheumatol 2009;36:8; doi:10.3899/jrheum.090200 